\title{
Possíveis contribuições do modelo homeopático à humanização da formação médica
}

\author{
Possible contributions of the homoeopathic \\ model to humanization of medical training
}

Marcus Zulian Teixeira ${ }^{\mathrm{I}}$

PALAVRAS-CHAVE
- Educação médica
- Homeopatia
- Currículo
- Humanismo
- Ética
- Bioética

KEY WORDS

- Education, medical

- Homeopathy

- Curriculum

- Humanism

- Ethics

- Bioethics

Recebido em: 26/11/2007 Reencaminhado em: 25/04/2008 Reencaminhado em: 19/08/2008 Aprovado em: 24/12/2008

\begin{abstract}
R E S U M O
Em virtude da valorização dos aspectos técnico-científicos no modelo biomédico, aspectos subjetivos da individualidade foram desprezados, tornando a medicina moderna desumanizada e reducionista na abordagem do processo de adoecimento humano. Com o intuito de resgatar a relação médico-paciente e a atenção integral ao indivíduo enfermo, com nítidos reflexos na qualidade e eficácia dos serviços prestados, a preocupação com a humanização da saúde passou a ser uma prioridade dos sistemas públicos, com a implantação de iniciativas nos diversos níveis de atenção. Desta forma, propostas humanísticas estão sendo incorporadas à formação médica nos cursos de graduação em Medicina e na rede de Atenção Básica em Saúde, visando contemplar, entre outros aspectos, "a compreensão dos determinantes sociais, culturais, comportamentais, psicológicos, ecológicos, éticos e legais, nos níveis individual e coletivo do processo saúde-doença”. Neste contexto, o modelo homeopático, por aplicar em sua prática clínica diária uma abordagem semiológico-terapêutica individualizante e humanística na compreensão do processo saúde-doença e no tratamento das enfermidades, valorizando os determinantes éticos, filosóficos, antropológicos, psicológicos e socioambientais, poderia contribuir de forma adjuvante e complementar a humanização da formação médica, desde que seus preceitos sejam incorporados de forma regular e gradativa ao processo ensino-aprendizagem das instituições públicas e acadêmicas.
\end{abstract}

\section{A B S T R A C T}

To the extent that technical and scientific aspects of the biomedical model assumed increasing importance, subjective characteristics of individuality were neglected, and modern medicine became dehumanized and narrow-minded in its approach to the processes involved in human illness. A current priority for government-run health systems is the determination to reclaim the physician-patient relationship, with comprehensive concern for patients and a clear effect on the quality and effectiveness of the services provided in an effort to achieve a more humane health system. Several new initiatives have been implemented at different levels of care. Humane propositions are thus being incorporated into medical training, in both undergraduate education and the public primary care system, with the aim of understanding the social, cultural, behavioral, psychological, environmental, ethical, and legal determinants of the health-disease process at the individual and community levels. In daily clinical practice, the homeopathic model applies a humane and individualized semiological-therapeutic approach to understanding the health-disease process and treatment of diseases, with attention to the value of ethical, philosophical, anthropological, and socio-environmental determinants and thus the potential for contributing positively to assisting and complementing medical education, as long as the model's principles are progressively incorporated into the teaching and learning process of public and academic institutions.

\footnotetext{
${ }^{I}$ Universidade de São Paulo, São Paulo, São Paulo, Brasil.
} 


\section{INTRODUÇÃO}

Embora os avanços da ciência contemporânea tenham trazido enormes progressos à medicina técnico-científica moderna, o modelo científico cartesiano, no intuito de aprofundar o estudo e o tratamento das partes constituintes do organismo de forma compartimentada, desprezou a relação médico-paciente e os aspectos subjetivos da individualidade enferma, tornando a medicina mecanicista, desumanizada e fragmentada na atenção integral ao paciente. Centrada num modelo farmacológico reducionista, que prioriza o restabelecimento funcional dos sistemas orgânicos de forma dissociada, ignorou os conhecimentos crescentes sobre a fisiologia integrativa (eixo psiconeuro-imuno-endócrino-metabólico), que reage às alterações medicamentosas isoladas da homeostase interna, produzindo novos distúrbios ou exacerbando manifestações antigas, ampliando o espectro das doenças crônicas.

Prova deste descompasso do modelo biomédico vigente é o interesse crescente da população e da classe médica em geral por práticas não convencionais em saúde, que valorizam os aspectos humanísticos, em busca de um incremento na relação médico-paciente e de uma terapêutica que se proponha a atuar de forma integrada na complexidade enferma (unidade corpo-mente-espírito), com menos efeitos colaterais do que os observados no tratamento alo-enantiopático convencional ${ }^{1-6}$.

Reiterando os resultados de outros levantamentos ${ }^{7-11}$, pesquisa realizada com 484 estudantes da Faculdade de Medicina da Universidade de São Paulo (FMUSP) ${ }^{12}$ evidenciou que mais de $85 \%$ dos entrevistados consideravam que a homeopatia e a acupuntura deveriam estar inseridas no currículo da graduação das escolas de Medicina (eletiva: 72\%; obrigatória: 19\%), com $56 \%$ mostrando-se bastante interessados no aprendizado. Ao redor de $35 \%$ dos acadêmicos foram favoráveis ao oferecimento ambulatorial das especialidades nos serviços públicos de saúde, enquanto a média de $34 \%$ defendeu a oferta destes tratamentos também em hospitais, com $60 \%$ acreditando na possibilidade de integração com a prática médica convencional.

Em 2001, a Comissão da Câmara de Educação Superior do Conselho Nacional de Educação aprovou as Diretrizes Curriculares Nacionais do Curso de Graduação em Medicina ${ }^{13}$, priorizando os aspectos humanísticos, éticos e da integralidade à saúde, esboçando o perfil desejado do médico recém-formado (Art. $3^{\circ}$ ):

O Curso de Graduação em Medicina tem como perfil do formando egresso/profissional o médico, com formação generalista, humanista, crítica e reflexiva. Capacitado a atuar, pautado em princípios éticos, no processo de saúde-doença em seus diferentes níveis de atenção, com ações de promoção, prevenção, recuperação e reabilitação à saúde, na perspectiva da integralidade da assistência, com senso de responsabilidade social e compromisso com a cidadania, como promotor da saúde integral do ser humano.

Com o intuito de resgatar estes fundamentos intrínsecos à verdadeira arte de curar, iniciativas no ensino médico e na atenção primária à saúde têm surgido ultimamente, buscando oferecer aos profissionais da saúde as ferramentas necessárias ao exercício humanístico da medicina.

Entendamos que toda prática médica será desenvolvida a partir de sua compreensão do processo de adoecimento humano, que pode variar desde a concepção integrativa entre os diversos aspectos da individualidade enferma (determinantes biopsicossocioambientais) até a visão reducionista dos aspectos orgânicos específicos. Em vista disto, a formação médica priorizará determinados fundamentos e atividades teórico-práticas em sua grade programática, a fim de capacitar o futuro médico nos requisitos mínimos para transmitir aos pacientes o retorno à saúde, segundo as concepções de saúde-doença ou normalidade-anormalidade propagadas.

Segundo as referidas diretrizes curriculares $\left(\text { Art. } 12^{\circ}\right)^{13}$, a estrutura do curso de graduação em Medicina deveria "incluir dimensões éticas e humanísticas, desenvolvendo no aluno atitudes e valores orientados para a cidadania", assim como "promover a integração e a interdisciplinaridade em coerência com o eixo de desenvolvimento curricular, buscando integrar as dimensões biológicas, psicológicas, sociais e ambientais". Apesar de bastante propagados e difundidos, estes parâmetros não são satisfatoriamente identificados no presente formato educacional ${ }^{14}$.

Neste contexto de ensino-aprendizagem, o modelo homeopático, por valorizar tradicionalmente aspectos humanísticos (éticos, filosóficos, antropológicos, psicológicos, sociológicos, ambientais, etc.) no entendimento e no tratamento das enfermidades humanas, poderia contribuir de forma adjuvante e complementar à humanização da formação médica ${ }^{15,16}$, desde que incorporado de forma regular e gradativa à educação médica, tanto na graduação quanto na rede de atenção primária à saúde.

\section{PROPOSTAS DE HUMANIZAÇÃO NA FORMAÇÃO MÉDICA}

Nas diversas propostas de humanização da formação médica, são reiterados aspectos específicos que destacam os requisitos mínimos e necessários para que as estruturas dos cursos de graduação em Medicina e dos serviços de Atenção Básica em Saúde possam responder a esta demanda humanística reprimida. 
São citados a seguir diversos textos, que enfatizam o núcleo temático das diretrizes propostas, que iremos relacionar adiante com os pressupostos da concepção homeopática.

Propondo uma abordagem ampla e integrativa dos múltiplos aspectos que podem interferir no processo saúde-doença, as iniciativas de humanização dos cursos de graduação em Medicina passam a valorizar a individualização do paciente na prática médica, associando aspectos psicossocioambientais aos aspectos biológicos do atual modelo biomédico:

A formação do médico tem por objetivo dotar o profissional dos conhecimentos requeridos para o exercício das seguintes competências e habilidades específicas: dominar os conhecimentos científicos básicos da natureza biopsicossocioambiental subjacentes à prática médica e ter raciocínio crítico na interpretação dos dados, na identificação da natureza dos problemas da prática médica e na sua resolução. (Brasil ${ }^{13}, 2001$, Art. 5º)

Os conteúdos essenciais para o Curso de Graduação em Medicina devem estar relacionados com todo o processo saúde-doença do cidadão, da família e da comunidade, integrado à realidade epidemiológica e profissional, proporcionando a integralidade das ações do cuidar em medicina. Devem contemplar: compreensão dos determinantes sociais, culturais, comportamentais, psicológicos, ecológicos, éticos e legais, nos níveis individual e coletivo do processo saúde-doença; abordagem do processo saúde-doença do indivíduo e da população, em seus múltiplos aspectos de determinação, ocorrência e intervenção; compreensão e domínio da propedêutica médica - capacidade de realizar história clínica, exame físico, conhecimento fisiopatológico dos sinais e sintomas; capacidade reflexiva e compreensão ética, psicológica e humanística da relação médico-paciente. (Brasil ${ }^{13}, 2001$, Art. 6º)

A estrutura do Curso de Graduação em Medicina deve: incluir dimensões éticas e humanísticas, desenvolvendo no aluno atitudes e valores orientados para a cidadania; promover a integração e a interdisciplinaridade em coerência com o eixo de desenvolvimento curricular, buscando integrar as dimensões biológicas, psicológicas, sociais e ambientais. (Brasil ${ }^{13}, 2001$, Art. 12º)
De forma análoga, o papel da rede de Atenção Básica em Saúde (ABS) na formação médica foi explicitado pela Associação Brasileira de Educação Médica, com o intuito de aprofundar a discussão dos fundamentos de integralidade, equidade e universalidade do Sistema Único de Saúde (SUS ${ }^{17}$. Entre as diretrizes apontadas para a capacitação médica na rede primária de atenção à saúde, foram destacados os aspectos facilitadores da humanização da medicina e da valorização de aspectos subjetivos da individualidade enferma:

Clínica ampliada: [...] Assim, a clínica deverá ser ampliada, partindo de seu núcleo biomédico para os aspectos subjetivos e sociais de cada sujeito, respeitando a característica singular de cada caso - "cada caso é um caso" - sem abrir mão de critérios técnicos previamente definidos (diretrizes clínicas, programas, etc.).

Modelo Organizacional para a ABS-Diretrizes: - Reformulação do saber e da prática tradicional em saúde: recomenda-se uma reformulação e ampliação do saber clínico, com a incorporação de conceitos e de ferramentas originários da saúde coletiva, saúde mental, ciências sociais e de outros campos do conhecimento que permitam aos trabalhadores de saúde lidar com a complexidade do processo saúde e doença, incorporando o social e o subjetivo, bem como fazer a gestão do trabalho em equipe e em sistemas de rede. [...] A Abordagem Integral depende da reformulação do paradigma tradicional denominado de biomédico.

Tanto nos cursos de graduação em Medicina quanto na rede de Atenção Básica em Saúde, a capacitação do médico nas diretrizes humanísticas está diretamente relacionada ao oferecimento de propostas educacionais e vivenciais que ampliem o entendimento dos aspectos éticos, filosóficos, antropológicos, sociológicos e psicológicos da natureza humana, fornecendo ferramentas que permitam diagnosticar e atuar na dinâmica multifatorial e complexa do adoecimento humano. Em busca desta mudança de paradigma, grupos de estudo e disciplinas ligados às áreas das humanidades médicas começam a ser constituídos nos ambientes acadêmico-institucionais ${ }^{18}$, incorporando à formação médica os aspectos humanísticos até pouco tempo desprezados pela rotina biomédica.

Como fator adicional, práticas médicas não convencionais que utilizem o modelo biopsicossocioambiental na compreensão e no 
tratamento das doenças humanas (homeopatia e acupuntura, por exemplo) poderiam auxiliar na "reformulação do saber e da prática tradicional em saúde"17, adicionando aspectos subjetivos à fisiopatologia objetiva do desequilíbrio orgânico, tornando a medicina uma ciência cada vez mais eficaz, efetiva e eficiente.

\section{CONTRIBUIÇÃO DO MODELO HOMEOPÁTICO À HUMANIZAÇÃO DA FORMAÇÃO MÉDICA}

O modelo homeopático surgiu em 1796 com Samuel Hahnemann, que propôs uma compreensão global e dinâmica do adoecimento humano e uma terapêutica que estimulasse o organismo a reagir contra seus próprios distúrbios. Administrando aos indivíduos enfermos substâncias que causam sintomas semelhantes em indivíduos sadios (experimentação patogenética homeopática), o medicamento homeopático tem o objetivo de despertar e direcionar uma reação homeostática curativa no sentido de neutralizar os desequilíbrios funcionais, emocionais, psíquicos, etc. que constituem a enfermidade natural. Ao longo da prática homeopática, Hahnemann diminuiu gradativamente as doses do medicamento homeopático, buscando minimizar os efeitos adversos indesejáveis que as substâncias poderiam causar nos pacientes quando empregadas segundo o princípio da similitude terapêutica (similia similibus curentur).

Especialidade médica reconhecida pelo Conselho Federal de Medicina (CFM) desde 1980, passou a ser oferecida em ambulatórios e centros de saúde da rede pública (SUS) a partir de 1985, contando, no Brasil, com aproximadamente 16 mil médicos praticantes. Segundo levantamento realizado pelo convênio Fiocruz/CFM ${ }^{19}$, a homeopatia, como especialidade principal de atuação, ocupava o décimo sexto maior contingente de profissionais entre as 61 especialidades analisadas.

Em relação à eficácia clínica do tratamento homeopático, questionada frequentemente pelos colegas de classe, três metanálises ${ }^{20-22}$ que utilizaram métodos de avaliação condizentes com a homeopatia evidenciaram efeitos superiores do tratamento homeopático geral frente ao placebo. Metanálises específicas evidenciaram a eficácia do tratamento homeopático frente ao placebo na influenza $a^{23}$, nas alergias ${ }^{24,25}$ e na diarréia infantil ${ }^{26}$. Ensaios clínicos placebo-controlados isolados evidenciaram a eficácia do tratamento homeopático individualizado na enxaqueca $^{27}$, na fibromialgia ${ }^{28}$, no transtorno do déficit de atenção e hiperatividade ${ }^{29,30}$ e na prevenção das infecções do trato respiratório superior ${ }^{31}$.

Apesar do desenvolvimento da pesquisa clínica na última década, a própria abordagem semiológico-terapêutica humanística e individualizadora do modelo homeopático - na qual indivíduos portadores de uma mesma doença podem receber medicamentos distintos segundo a totalidade de sintomas característicos que apresentem - impede a aplicação dos desenhos de en- saios clínicos clássicos na avaliação da eficácia do tratamento homeopático. São necessários modelos específicos e condizentes com a episteme homeopática que, muitas vezes, encontram barreiras em sua aplicabilidade no meio acadêmico e científico convencional ${ }^{32-34}$.

Por meio de citações do Organon da arte de curar ${ }^{35}$, que descreve os pressupostos fundamentais da racionalidade médica homeopática, iremos ilustrar alguns dos determinantes humanísti$\cos$ (éticos, filosóficos, antropológicos, psicológicos e socioambientais) implícitos ao modelo homeopático, evidenciando as contribuições que o aprendizado e a aplicação destes conceitos podem trazer à humanização da formação médica.

\section{Aspectos éticos do modelo homeopático}

Insatisfeito com as medicinas heróicas de sua época, que espoliavam as forças e os humores vitais do organismo com a aplicação de sangrias, sudoríferos, diuréticos, eméticos, laxantes etc., Hahnemann aplica os princípios bioéticos da beneficência e da não maleficência ao propor uma prática médica segura e menos iatrogênica, que visasse estimular as forças curativas do organismo com doses mínimas das substâncias medicinais:

[...] A homeopatia sabe que uma cura somente pode produzir-se através da reação da força vital contra o medicamento apropriado, sendo tal cura tanto mais segura e mais rápida quanto mais a força vital prevalecer no doente. Por essa razão, a homeopatia evita mesmo o mínimo enfraquecimento [...], servindo-se apenas de medicamento cujo poder de alterar e transformar o estado de saúde ela conheça exatamente, escolhendo um cujas forças modificadoras são capazes de remover a doença natural existente por sua semelhança com ela (similia similibus), simplesmente ministrando o mesmo em pequenas doses; conseqüentemente, a doença natural é extinta sem o mínimo debilitamento, sem martírios e sofrimentos e o doente, já durante a convalescença, fortalece-se, ficando, assim, curado [...] (Hahnemann ${ }^{35}$, 1995).

\section{Aspectos filosóficos do modelo homeopático}

Envolto pelo pensamento filosófico vitalista de sua época, Hahnemann atribui ao princípio vital o poder de regular, de forma automática e instintiva, todas as sensações e funções fisiológicas, desde que o organismo permanecesse no estado de saúde. O estado de doença estaria relacionado a um desequilíbrio desta força vital, e a saúde seria restabelecida com o reequilíbrio vital. Apesar de se tra- 
tar de uma terminologia em desuso pelo pensamento científico moderno, os fenômenos vitais, descritos por Hahnemann em função de suas observações clínicas, equivalem aos mecanismos homeostáticos estudados pela fisiologia moderna.

Desprezando e criticando qualquer especulação filosófica ou metafísica sobre a natureza desta força vital, Hahnemann encara o processo de adoecimento como um enfraquecimento dos mecanismos fisiológicos normais de adaptação e compensação. Ele correlaciona este desequilíbrio interior às diversas manifestações sintomáticas da individualidade, utilizando a totalidade de sinais e sintomas característicos do paciente como o único referencial para diagnosticar este padecimento da força vital (predisposição individual, suscetibilidade mórbida ou desequilíbrio homeostático) e para prescrever o medicamento homeopático mais semelhante à individualidade enferma.

\section{Aspectos antropológicos do modelo homeopático}

O modelo antropológico homeopático vitalista entende que o indivíduo humano é constituído por um princípio inteligente (mente ou consciência) que utiliza o corpo físico para cumprir seus propósitos de vida, mantendo sua saúde por meio do equilíbrio homeostático das sensações e funções fisiológicas (processo vital harmônico).

Empregando a totalidade de sinais e sintomas característicos da individualidade enferma para diagnosticar o desequilíbrio vital e, consequentemente, escolher o medicamento mais indicado segundo o princípio da similitude terapêutica, o modelo antropológico homeopático valoriza a integração das "dimensões biológicas, psicológicas, sociais e ambientais" ${ }^{\prime 13}$ na contextualização do adoecimento humano, antecipando os conceitos modernos sobre o papel da integração fisiológica (equilíbrio psiconeuro-imuno-endócrino-metabólico) no fenômeno saúde-doença:

Como auxílio à cura são úteis ao médico os pormenores acerca da causa mais provável da doença aguda, assim como os momentos mais significativos de toda a história clínica da doença crônica, a fim de descobrir sua causa fundamental [...], devendo ser levado em consideração a constituição física evidente do doente (principalmente do doente crônico), seu caráter com seu psiquismo e mente, suas ocupações, seus hábitos e modo de vida, suas relações sociais e domésticas, sua idade e função sexual, etc. ${ }^{35}(\S 5)$

[...] a totalidade de seus sintomas, esse quadro do ser interior da doença que se reflete no exterior
[...], deve ser o principal ou o único através do qual a doença dá a conhecer o meio de cura de que ela necessita, o único que pode determinar a escolha do meio de auxílio adequado - em suma, a totalidade dos sintomas deve ser, para o artista da cura, a coisa principal, senão a única que ele, em cada caso de doença, precisa conhecer e afastar através de sua arte, a fim de que a doença seja curada e transformada em saúde. ${ }^{35}(\S 7)$

\section{Aspectos psicológicos do modelo homeopático}

Perante esta totalidade de sintomas peculiares e característicos do indivíduo enfermo, a homeopatia prioriza os aspectos decorrentes do estado emocional e psíquico, por acreditar, segundo uma concepção psicossomática do adoecimento humano, que

em todas as doenças físicas, a disposição psíquica e mental está sempre se modificando e, em todos os casos de doença, que devem ser curados, o estado psíquico deve concorrer como um dos mais notáveis no conjunto característico dos sintomas, se quisermos traçar um quadro fidedigno da doença, a fim de poder tratá-la homeopaticamente, com êxito. ${ }^{35}(\S 210)$

Elegendo as características mentais e psíquicas como aspectos de alta hierarquia entre os demais sintomas do doente (que traduzem a ascendência do sistema límbico, centro das emoções, no equilíbrio homeostático), reforça a importância destas peculiaridades na escolha individualizada do medicamento homeopático e no correspondente êxito do tratamento, tanto para doenças crônicas quanto para doenças agudas:

Por conseguinte, jamais se poderá curar de acordo com a natureza, isto é, homeopaticamente, se não se observar, simultaneamente, em cada caso individual de doença, mesmo nos casos de doenças agudas, os sintomas das alterações mentais e psíquicas e se não se escolher, para alívio do doente, entre os medicamentos, uma tal potência morbífica que, a par da semelhança de seus outros sintomas com os da doença, também seja capaz de produzir por si um estado psíquico ou mental semelhante. ${ }^{35}(\S 213)$

\section{Aspectos socioambientais do modelo homeopático}

Na concepção global e individualizante do modelo homeopático, aspectos socioambientais são valorizados na compreen- 
são da gênese dos padecimentos humanos e na promoção à saúde, buscando-se eliminar as influências nocivas e prejudiciais (obstáculos à cura) ao equilíbrio natural dos pacientes e à atuação dos medicamentos homeopáticos:

São impropriamente chamadas de doenças os padecimentos dos indivíduos que constantemente se expõem a influências nocivas evitáveis, abusando habitualmente de bebidas ou alimentos nocivos; entregando-se a excessos de toda sorte que minam a saúde, suprimindo continuamente as necessidades básicas da vida; vivendo em regiões insalubres, sobretudo pantanosas; residindo em porões, oficinas úmidas ou em qualquer moradia sem ventilação, privando-se de movimento e de ar puro, arruinando sua saúde mediante excessivos esforços físicos e mentais; vivendo constantes dissabores, etc. Tais estados de falta de saúde, ocasionados pelas próprias pessoas, cessam espontaneamente e não podem levar o nome de doenças crônicas. ${ }^{35}(\S 77)$

A seguir, devem ser levados em consideração a idade do doente, seu modo de vida e de alimentação, sua situação doméstica, suas relações sociais, etc., a fim de verificar se esses elementos contribuíram para aumentar seu mal ou até que ponto poderão favorecer ou dificultar o tratamento. Igualmente não devem ser negligenciados seu psiquismo e sua maneira de pensar, a fim de saber se apresentam algum obstáculo ao tratamento ou se necessitam outra direção, psiquicamente serem estimulados ou modificados. ${ }^{35}(\S 208)$

\section{Aspectos humanísticos da semiologia homeopática}

A necessidade do conhecimento aprofundado dos sinais e sintomas particulares do doente, a fim de que se possa escolher o medicamento homeopático que tenha despertado semelhante totalidade de sintomas no experimentador sadio, impõe à prática homeopática, como premissa necessária e obrigatória, uma técnica semiológica que permita um exame minucioso dos diversos aspectos individuais (psíquicos, emocionais, físicos, sociais, familiares, ambientais, climáticos, alimentares, etc.). A importância do relato espontâneo do paciente e da investigação dos pormenores envolvidos no padecimento pessoal deve fazer do médico homeopata um observador imparcial, cuidadoso e interessado nas diversas particularidades do sofrimento humano:
O doente se queixa do desenvolvimento de seus males; as pessoas que o rodeiam relatam suas queixas, seu comportamento e o que perceberam nele; o médico vê, ouve e observa com os demais sentidos o que há nele de alterado ou fora do comum. Escreve exatamente tudo o que o paciente e seus amigos lhe disseram, com as mesmas expressões por eles utilizadas. Se possível, permanece em silêncio deixando-os falar sem interrompê-los, a menos que se desviem para outros assuntos (pois cada interrupção perturba o encadeamento do pensamento do narrador, posteriormente não lhe ocorrendo de novo tudo exatamente como ele pretendia dizer a princípio) $[\ldots] \cdot .^{35}(\S 84)$

Se durante estas informações espontâneas nada se mencionou em relação às várias partes ou funções do corpo ou acerca da disposição psíquica, o médico pergunta o que mais ele recorda que se relacione a estas partes e funções, assim como às condições psíquicas e mentais do doente, usando, porém, expressões gerais, a fim de que o informante seja obrigado a se expressar mais particularmente acerca de si [...]. ${ }^{35}(\S 88)$

Aspecto de fundamental importância para o esclarecimento das diversas suscetibilidades envolvidas no processo do adoecimento humano, a história biopatográfica - bio, vida + pathos, sofrimentos + graphos, registros -é parte integrante e fundamental da anamnese homeopática, constituindo um instrumento de grande valia na humanização do ato médico. Momento mágico da relação médico-paciente, o questionamento sobre os prováveis fatores desencadeantes (suscetibilidades psíquicas, emocionais, físicas, sociais, familiares, ambientais, etc.) do aparecimento ou da agravação da doença - exemplificado na resposta à pergunta: o que aconteceu ou mudou em sua vida antes do aparecimento ou da agravação da doença? - permite ao paciente entrar em contato com suas idiossincrasias e ao médico identificar aspectos fundamentais e particulares do complexo processo do adoecimento humano. Para o homeopata, a tradução destas suscetibilidades em sintomas facilita a escolha do medicamento individualizado segundo o princípio da similitude terapêutica.

\section{DISCUSSÃO}

Buscando resgatar uma relação positiva entre profissionais da saúde e usuários, com nítidos reflexos na qualidade e na eficácia dos serviços prestados, a preocupação com a humanização 
da saúde passou a ser uma prioridade dos sistemas públicos, com a implantação de propostas nos diversos níveis de atenção: Política Nacional de Humanização (PNH), HumanizaSUS, Programa Nacional de Humanização da Assistência Hospitalar (PNHAH), etc.

Assumindo papel fundamental neste processo, o médico deve estar preparado para responder a estas demandas humanísticas crescentes, sendo preciso que sua formação forneça subsídios para tal intento. São necessárias mudanças no paradigma biomédico vigente, tanto na valorização da relação médico-paciente quanto na compreensão integral do processo saúde-doença. A elaboração de políticas humanísticas, tanto no trabalho como na educação, é uma das prioridades prementes.

A ausência de condições de trabalho dignas - ambiente humanizado, remuneração e plano de carreira profissional satisfatórios, número de atendimentos e tempo de consulta compatíveis com a dinâmica humanística, etc. - dificulta a aplicação das propostas citadas, mesmo num profissional preparado e motivado para exercer a verdadeira arte de curar, permitindo o surgimento natural do impasse shakespeariano: ser ou não ser, eis a questão!

Centrados nos ideais da educação médica humanística, inúmeros educadores têm-se empenhado em elaborar e implantar propostas concretas de incremento das humanidades na grade curricular dos cursos de graduação em Medicina, pois quem sabe faz a hora, não espera acontecer.

Entre os diversos conteúdos éticos e humanísticos citados, vale ressaltar o diferencial que o paradigma homeopático, por meio da valorização da totalidade sintomática característica no entendimento e no tratamento integral da individualidade enferma, poderia acrescentar à "reformulação do saber e da prática tradicional em saúde, incorporando conceitos e ferramentas que permitam aos médicos lidar com a complexidade do processo saúde e doença" ${ }^{\prime 17}$. Priorizando em sua dinâmica semiológico-terapêutica a "compreensão dos determinantes sociais, culturais, comportamentais, psicológicos, ecológicos, éticos e legais do processo saúde-doença"13, permite "promover a integração e a interdisciplinaridade em coerência com o eixo de desenvolvimento curricular, buscando integrar as dimensões biológicas, psicológicas, sociais e ambientais"13.

Correspondendo aos anseios humanísticos da formação médica, disciplinas eletivas ou obrigatórias de homeopatia poderiam ser incorporadas ao currículo das faculdades de Medicina ${ }^{36}$, transmitindo aos estudantes algumas das "competências éticas e relacionais que todo aluno de Medicina deve adquirir ao longo da sua formação para se graduar como médico"18, ${ }^{\prime 18}$ por exemplificar por meio dos ensinamentos teórico-práticos muitos dos “ob- jetivos terminais da área de humanidades no ensino médico de graduação"18.

Além da valorização dos aspectos éticos, filosóficos, antropológicos, psicológicos e socioambientais já descritos, a aplicação da história biopatográfica homeopática, nem sempre valorizada na "história pessoal, social e familiar" da semiologia médica clássica, traria enormes benefícios à humanização da medicina, incrementando a relação médico-paciente sob diversos aspectos: ao médico, permitiria vislumbrar, de forma racional e repetitiva, a importância dos aspectos não fisiológicos (espirituais, emocionais, psíquicos, sociais, familiares, etc.) no despertar ou agravar das condições patológicas latentes, fazendo-o valorizar a semiologia subjetiva; ao paciente, estimularia o contato com suas próprias idiossincrasias, alertando-o para os meandros da dinâmica psicossomática e tornando-o copartícipe no processo de cura pessoal.

\section{CONSIDERAÇÕES FINAIS}

Assim como em séculos passados floresceram outros sistemas em medicina, no século 20 a medicina técnico-científica atingiu seu apogeu, aprofundando o estudo das ciências básicas em busca das causas fundamentais das doenças, finalizando seus dias com a promessa de que a terapia gênica seria a resposta para a cura das doenças crônicas, que se avolumam em variedade e quantidade nas últimas décadas. Dividindo o ser humano em partes para estudá-lo sistematicamente, desprezou os aspectos subjetivos da individualidade enferma, em vista da dificuldade de localizá-los nos segmentos fisiológicos humanos e mensurá-los segundo os padrões bioquímicos e os diagnósticos clássicos.

Acompanhando este processo, o ensino médico incorporou a imagem de que indivíduos doentes se assemelhariam a máquinas quebradas, priorizando o ensino compartimentado dos sistemas orgânicos e de suas funções como peças constituintes e suficientes para a manutenção da totalidade orgânica, e desprezando a integração fisiológica que evidencia a existência de um mecanismo homeostático regulador do processo saúde-doença, diretamente influenciado pelos aspectos subjetivos da individualidade humana.

No intuito de incorporar à biomedicina os aspectos humanísticos, melhorando a relação médico-paciente e ampliando a visão do processo de adoecimento humano, a proposta de humanização da formação médica atual se baseia na incorporação das disciplinas de humanidades nos cursos de graduação e na rede de Atenção Básica em Saúde.

Incrementando estas iniciativas, o ensino-aprendizagem dos aspectos teóricos e práticos do modelo homeopático nestes cenários possibilitaria aos pacientes ${ }^{37}$, estudantes de medicina ${ }^{38}$ e 
médicos do SUS ${ }^{39}$ vivenciarem a dinâmica de uma prática médica humanística por excelência, centrada na valorização do processo de adoecimento individual ${ }^{40}$ e numa proposta terapêutica que objetiva estimular o organismo a reagir contra seus próprios desequilíbrios, sem os inconvenientes dos efeitos colaterais das drogas clássicas.

Trabalhando de forma conjunta e interdisciplinar com as demais especialidades médicas do SUS, a homeopatia poderia contribuir satisfatoriamente para uma "clínica ampliada" e uma "reformulação do saber e da prática tradicional em saúde" ${ }^{17}$, evidenciando sua capacidade de minimizar inúmeros sofrimentos humanos crônicos e os altos custos do modelo biomédico vigente, cumprindo o pressuposto da integralidade ${ }^{41}$ na atenção em saúde. Neste contexto, os pressupostos da equidade e da universalidade também seriam satisfeitos, desde que fosse permitido o livre acesso da população ao tratamento homeopático.

Por intermédio destes cenários idealizadores do ensino e da prática médica acadêmico-institucional, a possibilidade de troca de conhecimentos e experiências entre estudantes, médicos, professores e pesquisadores homeopatas e não homeopatas permitiria minimizar os excessos de ambas as racionalidades, dirimindo preconceitos seculares ${ }^{38}$, contribuindo para o incremento da arte médica e permitindo que o espírito hipocrático, existente em todos nós, assuma seu verdadeiro valor.

\section{REFERÊNCIAS}

1. Furnham A, Forey J. The attitudes, behaviors, and beliefs of patients of conventional vs complementary (alternative) medicine. J Clin Psychol. 1994;50:458-69.

2. Astin JA. Why patients use alternative medicine: results of a national survey. JAMA 1998; 279:1548-53.

3. Veeramah EK, Holmes S. Complementary therapy: complement or threat to modern medicine? J R soc Health. 2000;120(1):42-6.

4. Linzer M, Konrad TR, Douglas J, McMurray JE, Pathman DE, Willians ES, et al. Management care, time pressure, and physician job satisfaction: results from the physician worklife study. J Gen Intern Med. 2000;15:441-50.

5. Haas JS, Cook EF, Puopolo AL, Burstin HR, Cleary, PD, Breannan. Is the professional satisfaction of general internists associated with patient satisfaction? J Gen Intern Med. 2000;15(2):122-3.

6. Teixeira MZ, Lin CA, Martins MA. O ensino de práticas não-convencionais em saúde nas faculdades de medicina: panorama mundial e perspectivas brasileiras. Rev Bras Educ Med. 2004;28(1):51-60.
7. Rampes H, Sharples F, Maragh S, Fisher P. Introducing complementary medicine into the medical curriculum. J R Soc Med. 1997;90(1):19-22.

8. Derr S, Shaikh U, Rosen A, Guadagnino P. Medical students' attitudes toward, knowledge of, and experience with complementary medicine therapies. Acad Med. 1998;73:1020.

9. Duggan K, Verhoef MJ, Hilsden RJ. First-year medical students and complementary and alternative medicines: attitudes, knowledge and experiences. Ann R Coll Physicians Surg Can. 1999;32:157-60.

10. Owen D, Lewith GT. Complementary and alternative medicine (CAM) in the undergraduate medical curriculum: the Southampton experience. Med Educ. 2001;35(1):73-7. Rosenbaum ME, Nisly NL, Ferguson KJ, Kligman EW. Academic physicians and complementary and alternative medicine: an institutional survey. Am J Med Qual. 2002;17(1):3-9.

11. Dantas F, Ribeiro CT. Atitudes de estudantes de medicina sobre práticas médicas heterodoxas no Brasil. Rev Bras Educ Med. 2002;26(2):99-104.

12. Teixeira MZ, Lin CA, Martins MA. Homeopathy and acupuncture teaching at Faculdade de Medicina da Universidade de São Paulo: the undergraduates' attitudes. Sao Paulo Med J. 2005;123:77-82.

13. Brasil. Ministério da Educação. Conselho Nacional de Educação.Câmara de Educação Superior. Resolução CNE/CES nº 4, de 07 de novembro de 2001. Institui diretrizes curriculares nacionais do curso de graduação em medicina. [online]. [acesso em: set. 2007]. Disponível em: http://portal.mec.gov.br/cne/arquivos/pdf/CES04.pdf.

14. Vieira JE, Lopes Júnior A, Basile MA, Martins MA. Identificação das Diretrizes Curriculares Nacionais do Curso de Graduação em Medicina pelos alunos da Universidade de São Paulo. Rev Bras Educ Med. 2003;27(2):96-103.

15. Teixeira MZ. Homeopatia: prática médica coadjuvante. Rev Assoc Med Bras. 2007;53(4):374-6.

16. Teixeira MZ. Homeopatia: prática médica humanística. Rev Assoc Med Bras. 2007;53(6):547-9.

17. Campos GWS. Papel da Rede de Atenção Básica em Saúde na Formação Médica - Diretrizes:documento preliminar abril de 2005. [online]. [acesso em:set. 2007] Disponível em:http://www.abem-educmed.org.br/pdf/doc_prof_gastao.pdf. 
18. Faculdade de Medicina da Universidade de São Paulo. Centro de Desenvolvimento de Educação Médica Prof. Eduardo Marcondes (Cedem). Humanidades \& Medicina. Disponível em: http://www.fm.usp.br/cedem/hum/disciplinas.php.

19. Machado MH, (coord.). Perfil dos médicos no Brasil: relatório final. Rio de Janeiro: Fundação Oswaldo Cruz; Conselho Federal de Medicina; Ministério da Saúde; Programa das Nações Unidas para o Desenvolvimento; 1996.

20. Kleijnen J, Knipschild P, ter Riet G. Clinical trials of homoeopathy. BMJ. 1991;302:316-23.

21. Linde K, Clausius N, Ramirez G, Melchart D, Eitel F, Hedges LV et al. Are the clinical effects of homeopathy placebo effects? A meta-analysis of placebo-controlled trials. Lancet. 1997;350:834-43.

22. Linde K, Melchart D. Randomized controlled trials of individualized homeopathy: a state-of-the-art review. J Altern Complement Med. 1998;4(4):371-88.

23. Vickers AJ, Smith C. Homoeopathic Oscillococcinum for preventing and treating influenza-like syndromes. Cochrane Database Syst Rev. 2000;(2):CD001957.

24. Wiesenauer M, Lüdtke R. A meta-analysis of the homeopathic treatment of pollinosis with Galphimia glauca. Forsch Komplementärmed.1996; 3:230-6.

25. Taylor MA, Reilly D, Llewellyn-Jones RH, McSharry C, Aitchison TC. Randomised controlled trial of homoeopathy versus placebo in perennial allergic rhinitis with overview of four trial series. BMJ. 2000;321(7259):471-6.

26. Jacobs J, Jonas WB, Jiménez-Pérez M, Crothers D. Homeopathy for childhood diarrhea: combined results and metaanalysis from three randomized, controlled clinical trials. Pediatr Infect Dis J. 2003; 22(3):229-34.

27. Straumshein P, Borchgrevink C, Mowinckel P, Kierulf $H$, Hafslund O. Homeopathic treatment of migraine: a double blind, placebo controlled trial of 68 patients. Br Homeopath J. 2000; 89(1):4-7.

28. Bell IR, Lewis DA, Brooks AJ, Schwartz GE, Lewis SE, Walsh BT, Baldwin, CM. Improved clinical status in fibromyalgia patients treated with individualized homeopathic remedies versus placebo. Rheumatology. 2004;43(5):577-82.

29. Frei H, Everts R, von Ammon K, Kaufmann F, Walther D, Hsu-Schmitz SF et al. Homeopathic treatment of children with attention deficit hyperactivity disorder: a randomised, double blind, placebo controlled crossover trial. Eur J Pediatr. 2005;164(12):758-67.
30. Frei H, Everts R; von Ammon K, Kaufmann F, Walther D, Schmitz SF et al. Randomised controlled trials of homeopathy in hyperactive children: treatment procedure leads to an unconventional study design: Experience with open-label homeopathic treatment preceding the Swiss ADHD placebo controlled, randomised, double-blind, cross-over trial. Homeopathy. 2007;96(1):35-41. DOI: 10.1016/j.homp.2006.11.004

31. Steinsbekk A, Fonnebo V, Lewith G, Bentzen N. Homeopathic care for the prevention of upper respiratory tract infections in children: a pragmatic, randomised, controlled trial comparing individualised homeopathic care and waiting-list controls. Complement Ther Med. 2005;13(4):231-8. Doi:10.1016/j.ctim.2005.06.007

32. Jonas WB; Kaptchuk TJ; Linde K. A critical overview of homeopathy. Ann Intern Med 2003; 138:393-9.

33. Teixeira MZ. Pesquisa clínica em homeopatia: evidências, limitações e projetos. Pediatria (São Paulo) 2008; 30:27-40.

34. Teixeira MZ. Ensaio clínico quali-quantitativo para avaliar a eficácia e a efetividade do tratamento homeopático individualizado na rinite alérgica perene [tese na internet]. São Paulo: Faculdade de Medicina da Universidade de São Paulo; 2009. [acesso em: ago. 2007]. Disponível em: http://www.teses.usp.br/.

35. Hahnemann S. Organon da arte de curar. 2.ed. Ribeirão Preto: Museu de Homeopatia Abrahão Brickmann, 1995.

36. Universidade de São Paulo. Faculdade de Medicina da Universidade de São Paulo. Disciplina Optativa "Fundamentos da Homeopatia". Disponível em: http://medicina.fm.usp.br/homeopatia/.

37. Monteiro DA, Iriart JAB. Homeopatia no Sistema Único de Saúde: representações dos usuários sobre o tratamento homeopático. Cad Saúde Pública [online] 2007;23(8):1903-12. DOI: 10.1590/S0102-311X2007000800017

38. Teixeira MZ. Homeopatia: desinformação e preconceito no ensino médico. Rev Bras Educ Med. 2007; 31(1):15-20. DOI: $10.1590 /$ S0100-55022007000100003

39. Galhardi WMP, Barros NF. O ensino da homeopatia e a prática no SUS. Interface Comum Saúde Educ. 2008;12(25):247-66. DOI: 10.1590/S1414-32832008000200003

40. Araújo EC. Homeopatia: uma abordagem do sujeito no processo de adoecimento. Ciênc Saúde Coletiva. 2008;13(supl.):663-71. DOI: 10.1590/S1413-81232008000700015

41. Santanna C, Hennington EA, Junges JR. Prática médica homeopática e a integralidade. Interface Comum Saúde Educ. 2008; 12(25):233-46. DOI: 10.1590/S1414-32832008000200002 


\section{CONFLITO DE INTERESSES}

Declarou não haver.

\section{ENDEREÇO PARA CORRESPONDÊNCIA}

Marcus Zulian Teixeira
Rua Teodoro Sampaio, 352 - cj. 128

Pinheiros - São Paulo

CEP.: 05406-000 - SP

E-mail: marcus@homeozulian.med.br 